\title{
How to more effectively determine what is true: The limits of intuition
}

Martin Kaefer, Nicolas Kalfa, Katherine W Herbst, Luke Harper, Goedele MA Beckers, Darius Bagli and Magdalena Fossum

\section{Summary}

The plethora of scientific data and explosion of published materials often leave it challenging to develop a clear and concise overview of many scientific topics. A number of factors may contribute to our misunderstanding. It is the focus of this article to describe primary reasons for failure to establish a clear, factual and functional understanding regarding scientific areas of inquiry.

Our view of the world is often, if not always, skewed. There are a number of aspects of modern life that heavily influence our perception of reality. Personal bias, of which there are many forms, play a large roll [1]. We tend to generalize based on our own limited experience, and this may lead to incorrect assumptions. In addition, the rapid accumulation of new information often leads to outdated facts. What was true yesterday may, in many cases, be replaced by what is discovered even a few months later. Remaining up to date requires constant vigilance. Compounding these issues is the bias of news and pseudoscientific sources to emphasize the dramatic, with the creation of fake news lying at its ultimate extreme [2]. All of these factors contribute to our inability to effectively create a clear understanding of reality. Dr. Hans Rosling, the late swedish professor of global health, points out that an

This is the author's manuscript of the work published in final edited form as:

Kaefer, M., Kalfa, N., Herbst, K. W., Harper, L., Beckers, G. M., Bagli, D., Fossum, M., \& ESPU research committee. (2020). How to more effectively determine what is true: The limits of intuition. Journal of Pediatric Urology, 16(4), 495-496. https://doi.org/10.1016/i.jpurol.2020.05.004 
understanding of these pervasive influences and avoiding an overdependence on intuition is necessary in order to fight our "devastating ignorance" [1].

Intuition is the ability to understand something immediately, without the need for conscious reasoning. This ability can enhance our efficiency in our professional and personal lives. The skill to intuitively understand the body language or facial expression of a patient can play an important roll in delivery of care. However, intuition can often be quite erroneous and lead to poor understanding and decisions. One illustrative example is the birthday paradox which poses the question "If there are 23 people in a room, what is the likelihood that two will have the same birthday?". Most individuals will intuitively respond with a low number and fail to understand that there is a greater than $50 \%$ chance of this occurring. The Monty Hall Paradox is another famous example of how our intuition can mislead us and result in poor decision making [3]. ${ }^{1}$

The anecdote to overdependence on our intuition is a clear method for positing the problem presented and a better understanding of statistics and probability. Mark Twain is famous for stating, "There are lies, damned lies and statistics". It can also be said that the later, assuming they are properly applied, may often save us from the first two. This leads us to perhaps the most pervasively misunderstood aspect of this valued topic: the P-value.

The P-value, a value that is derived from testing the null hypothesis, helps us understand the likelihood that there is an association between variables. ${ }^{2}$

\footnotetext{
${ }^{1}$ For an understanding of this intriguing paradox the reader is referred to the websites https://www.khanacademy.org/math/precalculus/x9e81a4f98389efdf:probcomb/x9e81a4f98389efdf:dependent-events-precalc/v/monty-hall-problem and http://www.shodor.org/interactivate/activities/SimpleMontyHall/
}

\footnotetext{
${ }^{2}$ In fact, a more pure statement would be that the $P$ value indicates the degree to which the data conform to the pattern predicted by the test hypothesis if all assumptions (i.e. data collection, adherence to protocols, etc.) were true.
} 
Sir Ronald Fisher of Fisher's exact test fame, proposed that a value of 0.05 was a reasonable threshold [4]. He arrived at this threshold because this would be at the boundary of two standard deviations if the data were evenly distributed in a bell shaped curve. If we employ this threshold, then it is generally believed that there is essentially a $5 \%$ chance that the perceived relationship is actually due to chance. However, two points need to be made quite clear in order to make reasonable use of this information. First, labeling results as statistically significant (or not) does not in fact tell us that there is a true relationship between variables. The P-value only gives us information as to the likelihood that it is true. Second, it would not seem appropriate to use the same threshold for all things considered. A threshold value of 0.05 may seem adequate when testing whether a relatively benign medication adequately treats prepucial adhesions. However, is this degree of confidence adequate for evaluating the relative safety of two operations when one procedure may carry the risk of a devastating and irreversible injury to the penis? Conversely, if data regarding a potentially life saving drug for a previously incurable disease yields a P-value of only 0.1 should we completely abandon use of the drug? Professional societies and experts play a vital role in understanding the impact of the data and hence play a vital role in determining what P-values (as well as other statistical parameters) are reasonable and adequate to expect.

It should be the foremost goal of all physicians and scientists to see the world more clearly. To this end we need to strive for a fact based world-view that is untainted by our own personal bias, overdependence on intuition, or misunderstanding of scientific methodology. It is only through a clear understanding that we will have a reasonable chance of confronting the challenging questions facing our profession. 


\section{Authors (On behalf of the ESPU research committee)}

Martin Kaefer (Riley Hospital for Children, Indiana University, Indianapolis, IN, United States)

Nicolas Kalfa (Service de Chirurgie Urologique Pe'diatrique, Ho^pital Lapeyronie, CHU de Montpellier, Universit'e de Montpellier, France)

Katherine W. Herbst (Division of Urology, Department of Research, Connecticut Children's Medical Center, Hartford, CT, United States)

Luke Harper (Service de Chirurgie Pe'diatrique, Ho^pital Pellegrin-Enfants, CHU de Bordeaux, France)

Goedele M.A. Beckers (Department of Urology, Pediatric Urology Section, Amsterdam University Medical Centre Location VUmc, Amsterdam, the Netherlands)

Darius Bagli (Division of Urology, Departments of Surgery and Physiology, University of Toronto, Developmental and Stem Cell Biology, The Hospital for Sick Children and Research Institute, Toronto, Ontario, Canada)

Magdalena Fossum (Department of Pediatric Surgery, Surgical Clinic C, Rigshospitalet, Copenhagen University, Copenhagen, Denmark; Department of Women's and Children's Health, Karolinska Institutet, Stockholm, Sweden) 


\section{References}

1. Rosling H., Rosling O., Rönnlund A.R.: Factfulness : ten reasons we're wrong about the world--and why things are better than you think,.1st ed.2018.Flatiron BooksNew Yorkpp. 342. pp. $x$

2. Harper L., Herbst K.W., Bagli D., Kaefer M., Beckers G.M.A., Fossum M., et. al.: The battle between fake news and science. J Pediatr Urol 2019; 16: pp. 114-115.

3. Farlow S.J.: Paradoxes in mathematics.2014.Dover PublicationsMineola, New Yorkpp. 168. pp. x

4. Fisher R.A.: Statistical methods for research workers.1925.Oliver and BoydEdinburgh, Londonpp. I. pp.

ix 